\title{
TTR
}

Traduction, terminologie, re?daction

\section{Traduire le texte dans son projet. Le littéralisme est soluble dans la poésie moderne : Auden}

Annie Brisset

Volume 12, numéro 2, 2e semestre 1999

Poésie, cognition, traduction II - Autour d'un poème de W. H. Auden Poetry, Cognition, Translation II — On a Poem by W. H. Auden

URI : https://id.erudit.org/iderudit/037370ar

DOI : https://doi.org/10.7202/037370ar

Aller au sommaire du numéro

Éditeur(s)

Association canadienne de traductologie

ISSN

0835-8443 (imprimé)

1708-2188 (numérique)

Découvrir la revue

Citer cet article

Brisset, A. (1999). Traduire le texte dans son projet. Le littéralisme est soluble dans la poésie moderne : Auden. TTR, 12(2), 39-56.

https://doi.org/10.7202/037370ar
Résumé de l'article

Traduire le texte dans son projet. Le littéralisme est soluble dans la poésie moderne : Auden - Bien qu'il soit reconnu comme l'un des plus grands poètes du XXe siècle, W. H. Auden est peu traduit en français. Son oeuvre, il est vrai, résiste à la traduction lorsqu'elle s'amuse, comme dans le poème qui sert ici d'exemple, à crypter le langage de tous les jours en le coulant dans une forme contrainte par la tradition. "The Three Companions » est un poème clé dans l'oeuvre d'Auden qui le publie au début des années trente et le reconduit dans plusieurs de ses recueils. Nous en proposons une analyse qui ouvre un dialogue avec chacune des traductions (une dizaine) et avec les commentaires qui les accompagnent.
Tous droits réservés @ C TTR: traduction, terminologie, rédaction — Les auteurs, 1999
Ce document est protége par la loi sur le droit d'auteur. L'utilisation des services d'Érudit (y compris la reproduction) est assujettie à sa politique d'utilisation que vous pouvez consulter en ligne. 


\title{
Traduire le texte dans son projet. Le littéralisme est soluble dans la poésie moderne : Auden
}

\author{
Annie Brisset
}

Le hasard d'un mot inconnu dans "The Three Companions" a mis Auden sur ma route. Non, je ne savais pas ce que "midden " voulait dire. Dans quel contexte? La question était bien trop naïve pour ce poème écrit, pourrait-on dire, contre ceux qui ont réponse à tout. Mais toute énigme contient la promesse d'une solution. Il suffirait d'analyser. Auden, poète pince-sans-rire, me précipita dans la sémiotique. Comprendre a besoin d'explication. La sémiotique, dit Ricour, est l'avers de l'herméneutique. La sémiotique jargonne? C'est vrai, mais on a vu la phobie du métalangage faire piétiner l'étude de la traduction, conduire à une herméneutique du n'importe quoi. Ce poème, dans sa curiosité excitant la mienne, a tout de même produit un exercice d'école. Je veux dire scolaire, car je n'arrivais pas à franchir l'obstacle de sa traduction. Tout cet arsenal d'analyse pour produire une mocheté pareille! On ne dissèque pas l'oiseau pour connaître son chant, dit Brodsky, il faut disséquer l'oreille qui écoute. Entre-temps, j'ai sollicité de grands traducteurs, j'ai sollicité des poètes. L'ironie mathématicienne de Jacques Roubaud, oulipien et anglophile, me semblait recouper les jeux langagiers d'Auden, auteur de ballades et de limericks. Mais Roubaud était en deuil et n'a pas répondu. Michel Deguy se montra intéressé, sans donner suite. Le poète portugais Graça Moura, traducteur de La Divine Comédie, ainsi que Didier Coste, romancier, poète, théoricien de la littérature et traducteur lui-même de quelquesuns des grands poètes espagnols, eurent chacun la gentillesse de m'envoyer leur version. Pierre Leyris dont j'admirais les traductions d'Hopkins me fit répondre par Antoine Berman : «Ce n'est pas "un moment" qu'il faudrait pour traduire ou même pour transposer systématiquement ce poème : l'éternité n'y suffirait pas pour la bonne raison qu'à chaque vers il se verrouille plus jalousement dans sa langue natale. Ta Canadienne m'a l'air d'être une sadique Gorgone à laquelle je tends le bouclier de l'Intraduisible pour qu'elle s'y mire et se pétrifie elle-même ". Rencontré à Montréal, Haroldo de Campos m'a inspiré par sa propre pratique la formule d'un atelier de traduction qui réunirait poéticiens, spécialistes de littérature anglo-américaine, traducteurs et 
poètes. Le projet s'est réalisé en 1998, à l'occasion du colloque "Poésie, cognition, traduction » organisé à l'Université d'Ottawa par l'Association canadienne de traductologie. Avec le concours additionnel de l'Association des traducteurs et traductrices littéraires du Canada et de plusieurs éditeurs de poésie, nous avons pu former un atelier ouvert au public.' De ce travail collectif, limité par le temps, sont issues les traductions que nous publions ici, accompagnées le plus souvent du commentaire de leurs auteurs. Ces traductions posent diverses questions. Parmi ces questions, en voici une qui me paraît essentielle : traduire la " lettre » est devenu un mot d'ordre au nom du respect de l'altérité du texte étranger, mais un dispositif d'écriture comme celui du poème d'Auden peut-il résister à la thèse littéraliste? Autrement dit, dans le procès de traduction n'y a-t-il pas lieu de distinguer entre le texte et son projet?

\section{Antécédence et intertexte du poème}

"The Three Companions " apparait pour la première fois en 1932 à la fin du recueil The Orators. Auden n'a pas encore donné de titre à ce poème qu'il présente alors comme "Épilogue » (titre retenu par Charlotte Melançon). Mais il le reprendra dans des anthologies successives en le joignant d'abord à d'autres poèmes désignés comme chants ou chansons ("Songs ", "Five Songs ") avant de lui donner son titre définitif. Chose certaine, en reconduisant ce poème de livre en livre, Auden lui marque un attachement particulier. ${ }^{2}$

Le lecteur entre de plain-pied dans le poème, une ballade qu'il reconnaît à son ouverture familière - " "O where are you going?", said so-and-so to so-and-so ». Ce topos est celui de la ballade du folklore anglais (sans rapport avec son homonyme français), qui prend aussi la forme de chanson à répondre. Le rythme, guilleret au premier abord, est créé par le mètre à quatre temps forts où se fixent les sons redoublés, les allitérations et les paronomases. La structure en écho

1 La table ronde réunissait Paul Bensimon, Serge Gavronsky, Patricia Godbout, Christine Klein-Lataud, Charlotte Melançon et Pierre Laurette. D'autres se sont joints à la discussion : Barbara Folkart, Clara Foz, Maurice Pergnier, Inês Oseki-Dépré, Jean Delisle...

Cet atelier a été rendu possible grâce à l'appui conjugué des services culturels de l'ambassade de France et de l'Alliance française. Les éditions du Vermillon, Le Nordir, Les Écrits des Hautes Terres et les éditions David ont aimablement prêté leur concours à l'événement.

2 Après sa publication initiale en épilogue du recueil The Orators (1932), le poème a successivement paru dans les anthologies suivantes: Selected Poems (Londres, Faber, 1938); Some Poems (Londres, Faber, 1938); Collected Poetry (New York, Random, 1945) où il figure dans la section intitulée "Songs "; Collected Shorter Poems 1930-1945 (Londres, Faber, 1950): il est inclus dans un groupe intitulé "Five Songs"; W. H. Auden: A Selection by the Author (Londres, Penguin, 1958) : Auden l'intitule « The Three Companions ». 
coïncide avec celle du dialogue : demanda celui-ci, répondit celui-là. Mais l'univers de la ballade médiévale est un univers allégorique et surnaturel, peuplé de figures inquiétantes. La forme du poème est modelée sur celle de la chanson "The Cutty Wren" ("O where are you going? said Milder to Malder... »). C'est une " vieille chanson de révolte paysanne ", écrit Arnold Wesker, qui l'utilise dans une de ses pièces pour dramatiser la révolte des soldats contre leurs officiers. ${ }^{3}$ Cette chanson qu'Auden connaissait puisqu'il a choisi de la faire figurer dans une de ses anthologies de la poésie anglaise appartient aussi à la tradition des chants de Noël, comme le rappelle Patricia Godbout.

Le récit allégorique du poème d'Auden a une antécédence qui remonte au Moyen Agge européen. Citant Le dit des trois morts et des trois vifs et La leggenda dei tre morti e dei tre vivi, Pierre Laurette rappelle à son tour qu'on retrouve « le thème des trois morts et des trois vifs chez Beaudoin de Condé, chez Nicolas de Margival, même thème en Allemagne, en Angleterre et en Italie aux $\mathrm{XIII}^{e}$ et XIV ${ }^{e}$ siècles ". Ce topos résonne chez Auden (chez Yeats également) dans plusieurs poèmes, par exemple "The Quarry ", dont l'imagerie fait écho à celle de "The Three Companions ", ou encore la ballade qui a pour refrain "Cried the cripples to the silent statue,/The six beggared cripples" (Hoggart, 1961, pp. 143-145), les trois mendiants étant par ailleurs associés à ce même topos.

La lecture du poème produit, dans les termes que Walter Benjamin applique à la poésie de Baudelaire (poète admiré par Auden) un "effondrement de l'aura ${ }^{4}$, un choc dialectique entre une forme conviviale, qui invite le lecteur à entrer dans la danse, et l'univers inquiétant qui en fait immédiatement le protagoniste d'une danse macabre. Tout ce qui va par deux et crée le tempo vivifiant va aussi par trois, le chiffre de la mort. Trois quatrains déroulent un questionnement auquel répondent les trois premiers vers de la dernière strophe. Trois paires de protagonistes et trois autres séries de paronomases (midden/madden // looking/lacking // swiftly/softly) ainsi que les rimes internes (furnaces/burn/return // path/pass/grass // see/trees/disease) soulignent la structure ternaire du poème. Cette architecture sous-tend un univers métaphysique tel qu'il est représenté dans l'iconographie médiévale, celle de Dürer par exemple. On se rappelle la gravure intitulée " Ritter, Tod und Teufel " (1513) où l'on voit un cavalier en

3 La ballade est entièrement reproduite avec sa partition musicale dans « Chips with Everything ", 1976, pp. 327-328. A. Wesker s'est encore servi du topos introductif de la ballade pour sa nouvelle «Said the old man to the young man ", 1978, pp. 27-77.

4 Walter Benjamin (1979 [1955]) applique le terme d'« aura " à l'ensemble des images qui, surgies de la mémoire involontaire, vont se cristalliser autour d'un objet. 
armure, lance au poing, cheminer de conserve avec la mort et le diable dans une gorge rocailleuse hérissée d'arbres morts, et tout au fond sur un promontoire les tours de la ville.

L'allégorie est le ressort premier de l'univers représenté par Auden. Dans une allégorie, chaque personnage incarne la série d'attributs attachés à son nom. Ici, la forme paranomastique qui les réunit en couples (reader/rider // fearer/farer // hearer/horror) souligne que les personnages sont unis dans un rapport dialectique, en accord avec le principe de la connaissance par les contraires.

\section{L'allégorie des personnages}

L'étymologie, qui joue un grand rôle chez Auden, fournit certaines clés. " Reader " vient du vieil anglais readan qui a donné riddle (d'où la figure du lecteur déchiffreur de l'énigme du poème) et signifie discerner, conseiller, en plus de lire. Le terme s'oppose à "rider» dérivé de ridan qui a donné road, le chemin et la route. "Rider», le cavalier, représente alors la bravoure - ce qui le rattache au chevalier (désigné par Ritter en allemand). C'est l'homme d'action confronté à l'intellectuel. Il incarne le mouvement contre l'immobilisme, le progrès contre les forces conservatrices. La sonorité est trop importante dans ce poème pour ne pas voir que le couple reader/rider recouvre un autre couple reader/writer qui reproduit la même dualité entre passif et actif et dont les termes sont à la fois complémentaires et permutables de part et d'autre d'une ligne qui, dans le poème, délimite les lieux de la connaissance.

Le couple fearer/farer décline la même opposition au moyen de traits sémantiques voisins. "Fearer", de faer qui a donné fare, incarne une inhibition d'ordre affectif, mais néanmoins fondée sur une rationalisation : j'ai peur parce que je sais d'expérience ou par le travail de mon imagination. À la pusillanimité de "fearer" s'oppose le goût de l'aventure et du risque associé à celui qui voyage, "farer", le pèlerin.

Dans le troisième et dernier couple allégorique, " horror » est celui que la peur extrême pétrifie, empêche à la fois de penser et d'agir. Il est contrasté avec " hearer », mot dérivé du vieil anglais hyran ou heran (hearken, hark). " Hearer " représente celui qui écoute et ainsi reçoit la connaissance. Il incarne l'obéissance, la soumission, la subordination.

Chacune des deux catégories de personnages fait apparaître une gradation. Du côté de l'action, «rider » est l'homologue de " farer », mais l'action qui les caractérise diminue d'intensité jusqu'à la troisième strophe où elle atteint son degré zéro chez " hearer», celui qui écoute et ne bouge plus. Inversement, " reader », celui qui raisonne 
sur les dangers en puissance, manifeste le degré initial et neutre de la peur qui gagne " fearer » et qui atteint son paroxysme chez « horror ».

En comparant les deux catégories de personnages, on voit que l'inaction culmine en même temps que la peur. Observation banale, si ce n'est que les personnages se révèlent interchangeables dans leur paradigme respectif, tout comme ils sont permutables à l'intérieur du couple qui les réunit. Au total, ils ne sont plus que deux au lieu de six. Chaque strophe peut donc se lire comme la mise en scène d'un état différent d'une même situation. Ainsi progresse le récit.

\section{Le logos du poème}

Dans la première strophe, " reader » emploie la raison sous la forme de trois arguments aux structures paralleles pour dissuader " rider» de partir :

That valley is fatal when furnaces burn

Yonder's the midden whose odours will madden

That gap is the grave where the tall return

À l'homme de réflexion, à celui qui analyse les raisons de ne pas bouger, l'homme d'action répondra par une phrase elliptique, lapidaire, conforme à ce qu'il représente : "Out of this house » - rien ne m'arrêtera, je pars quand même.

Le débat de la raison contre l'action se répète dans la seconde strophe. La topographie du lieu de l'entretien reste la même: les problèmes sont (allégoriquement) envisagés de haut. Mais le danger est désormais plus proche : le malheur concentré dans la vallée lointaine (" yonder ») est à moins d'une journée de marche. S'il part, "farer» atteindra le col à la tombée de la nuit et risque la chute dans un précipice (" the lacking ») qu'il n'aura pu voir. L'homme d'action fait au raisonneur la même réponse, avec la même économie de paroles : «Yours never will» - cela ne risque pas de t'arriver, sousentendu : je pars quand même.

Le récit s'accélère dans la troisième strophe. La nuit tombe. "What was that bird? " évoque l'" oiseau de Minerve » qui s'envole au crépuscule. On ne distingue plus que des formes (" shape »/" figure ») au milieu d'une forêt - archétype de la sécurité, comme la maison (" this house »), mais aussi tombeau végétal (G. Durand, 1963). Au début du poème, le danger était hypothétique. Il est devenu réel au point de se matérialiser, ici et maintenant, sous la forme d'une tache, signe de maladie (" the spot on your skin is a shocking disease "). Pour la troisième fois, la réponse est brève, elliptique ( $~$ They are looking for you »). Mais cette fois-ci, elle inverse les rôles en renvoyant au "lecteur » le reflet de sa fausse conscience. La réponse résonne comme 
un avertissement avant de déboucher sur l'action ("As he left them there $»)$.

J'ai évoqué le principe de la connaissance par les contraires. « Reader »/《 rider » l'homme de raison et l'homme d'action incarnent les deux versants complémentaires, l'esprit et le corps, qui définissent l'humain. Non plus deux, mais un seul et même protagoniste qui rassemble tous les autres : l'homme dans sa dualité, mais aussi dans la multiplicité, la répétitivité de ses comportements. La même opposition se dissout entre " reader 》 et "writer » et surtout entre « reader » et " hearer ". Moi, lecteur, interpellé par le poète, je deviens celui qui écoute les propos qu'il me prête ("said reader»). La fable qui m'est racontée est une maïeutique, la représentation d'un monologue intérieur (à la manière du délibéré qui caractérise le personnage cornélien). Auden estime que dans l'acte de lecture, le texte devient l'autre (" the other $\gg$ ) dans un dialogue auto-réflexif conduit par le lecteur. À propos de New Year Letter, le critique P. Deane observe que le lecteur est mis dans la position du locuteur : " that adduction must also to some extent involve a translation, of the text by the reader but also of the reader by the text » (1991, p. 183). Il cite encore Auden (1968, p. 126) pour qui tout dialogue est un fait de traduction, où les sujets échangent leur place respective. Cela entraîne plus exactement, précise le critique, une modification réciproque de leur position: « How profoundly appropriate, one reflects, was Auden's original title for the whole volume - The Double Man» (1991, p. 183). "The Three Companions " représente cette transformation au moyen d'une alternance dialogique qui place le lecteur en position (paradoxale) de parole (" said reader ») et par conséquent d'écoute (" to hearer »), puis à nouveau en position de parole ("said hearer "). Si toutefois les positions énonciatives sont réversibles par définition, l'échange correspond bien ici à un renversement de perspective : le lecteur tenté par l'immobilisme passe dans le camp de l'action. Auden déjoue ainsi le topos de la vie et de la mort, par un doublement dialectique de l'une par l'autre, source d'indétermination sémantique.

\section{Le paysage du poème : dialectique d'invitation et d'exclusion}

L'espace est le premier terme du poème, inscrit dans la question - «O where are you going? ". Le lieu d'où l'on parle, un lieu de savoir, un lieu sûr, mais celui de l'immobilité (du locuteur) ou de l'immobilisation (de l'interlocuteur) est opposé à un espace lointain où le danger existe en puissance (fatal when furnaces burn/the midden whose odours will madden). À l'opposition entre le proche et le lointain se superpose une autre opposition, entre le haut - état supérieur que procure la connaissance - et le bas, décrit en termes bibliques et qui incarne la mort, la décomposition des corps et la déchéance des facultés mentales. 
Dans la deuxième strophe, le thème de la chute se fait explicite. L'espace est paradoxalement déterminé par ce qui l'occulte dans la mesure où le temps qui doit s'écouler avant le crépuscule établit la distance entre le lieu d'où l'on parle et le lieu du danger. La tombée du jour marque le moment de la rupture entre la connaissance (la clairvoyance) et la cécité, entre la matière inerte et solide et la matière mouvante qui cède sous le pas et vous engloutit - « the lacking your footsteps feel from granite to grass » :

La dialectique du dur et du mou commande toutes les images que nous nous faisons de la matière intime des choses. Cette dialectique anime - car elle n'a son véritable sens que dans une animation toutes les images par lesquelles nous participons activement, ardemment, à l'intimité des substances. Dur et mou sont les premiers qualificatifs que reçoit la résistance de la matière, la première existence dynamique du monde résistant. Dans la connaissance dynamique de la matière - et corrélativement dans les connaissances des valeurs dynamiques de notre être rien n'est clair si nous ne posons pas d'abord les deux termes dur et mou. [...] La dureté et la mollesse des choses nous engagent - de force - dans des types de vie dynamique bien différents. Le monde résistant nous promeut hors de l'être statique, hors de l'être. [...] dans l'ordre de la matière, le oui et le non se disent mou et dur. Pas d'images de la matière sans cette dialectique d'invitation et d'exclusion, dialectique qui s'inversera parfois sous l'action de curieuses ambivalences jusqu'à définir, par exemple, une hostilité hypocrite de la mollesse ou une invite agaçante de la dureté. (Bachelard, 1948, pp. 17-18)

En même temps que le danger se rapproche, l'espace se rétrécit. À l'étalement de la vallée succède l'étroitesse d'un passage dans la montagne. La troisième strophe se déroule dans un espace sans déclivité qui s'est resserré autour des protagonistes désormais encerclés, piégés par un danger dont la présence indéfinissable finit par se concrétiser sous la forme d'une tache sur la peau. Pour le raisonneur incarné tour à tour par la figure allégorique du lecteur, de la peur et de l'horreur, le danger vient toujours de l'extérieur : c'est l'interlocuteur (" hearer ") qui se trouve soudain frappé par la maladie, contaminé par le milieu qui l'entoure. Le lieu maléfique, jusqu'alors associé à un futur hypothétique, a rejoint le lieu de la sécurité présumée et du présent immédiat.

Dans la progression du poème, la nature du danger se conjuge au lieu de sa manifestation pour instaurer une perspective dont le point de fuite est celui qui écoute - le lecteur effectif. Le poème réalise de cette façon une mise en abyme du logos. Dans le message adressé par le poète s'emboîte la série des épisodes de la fable que le lecteur, nommément désigné, est mis en demeure de déchiffrer. Seulement, le message demeure encrypté dans le poème. Il reste indécidable. On peut même dire que le renversement de perspective induit par le dernier vers de la troisième strophe, qui assimile « reader » et « hearer », constitue 
ce qu'en informatique on appelle une boucle. La boucle du poème ramène le lecteur à lui-même. Cela vaut pour l'écrivain (writeririder), pour le poète qui au lieu de se poser en donneur de leçon s'inclut dans ce qu'il donne à entendre. ${ }^{5}$ Si ce n'est que par ce dispositif le poème se défend justement contre le décryptage.

Tentons quand même une explication. Le poème met en scène les tensions existentielles d'une conscience individuelle partagée entre le statu quo et la nécessité d'agir, entre la crainte du changement et le désir d'une vie nouvelle rompant avec un univers figé, inscrit dans la mort. C'est une conscience divisée entre les forces inhibantes de la raison et les forces agissantes du corps qui poussent à la réalisation des impulsions et des désirs :

Authentic life has always been the goal of Auden's quest, and under this heading, shaping the theme of old life versus the new, the features of the early work gain meaning [...] those denounced as the " enemy " are representatives of the old and its willing perpetrators; it is the decadence of this life that is objectified in landscape, and freedom from its oppressive hold lies across the frontier. The central metaphor vindicating new allegiances is that of disease : the old life is diseased, the new life represents health. [...] Health demands change and growth, it demands, precisely, evolution; and the old order has grown rigid. (Greenberg, 1968, p. 19)

Ce débat est sous-tendu par le mythe de la chute dialectiquement représenté puisque l'espace dont on parle est, selon le point de vue (celui de "reader » ou celui de "rider»), enfer ou terre promise, ou du moins lieu de rédemption. Pour la voix de la raison et de la connaissance, l'être d'instinct est entaché, voué à la déchéance et promis à tous les tourments. Pour la voix de l'action, celle de la liberté, le fait d'être brimé dans la réalisation de ses désirs est source de maladie. L'individu (" he ») est par ailleurs opposé à un collectif (" them ॥) possédant des attributs qui permettraient de l'identifier avec les intellectuels, la bourgeoisie, les éducateurs ou l'ordre établi. L'individu remet en cause une société figée, dotée d'une fausse conscience, installée dans une fausse sécurité. Le recueil où paraît le poème pour la première fois porte en sous-titre An English Study. On a dit qu'il était le plus anglais des livres d'Auden :

The theme is, as usual, the diagnosis of symptoms and the prescription of a cure, in answer to the question stated in the opening prose poem "Address for a Prize Day » : "What do you think of England, this country of ours where no one is well? " There follows a destructive analysis of the golf-playing, church-going, tea-drinking,

5 « [...] righteousness wasn't Auden's character, nor would it escape him that he was part of the decade himself. A man like him wouldn't employ a negative epithet without sensing a touch of self-portrayal in it. " (Brodsky, 1986, p. 315) 
Boy Scoutish, eccentric English middle class, in which Auden scoffs at the self-love, frustration, inhibitedness, and so on, of schoolmasters, parsons, spinsters, and gentry. (Spender, 1964, p. 29)

Mais le poème doit également se lire dans un contexte moins immédiatement lié à l'Angleterre. The Orators paraît une dizaine d'années après la prise du pouvoir par Mussolini et juste avant l'avènement d'Hitler : " The effect of the turmoil of the 1930's on Auden's poetry was a tremendous inflow of new impressions, influences and ideas, which he met with an ever-increasing virtuosity [...]. The 1930's were a perpetual state of emergency for those aware that there was an emergency. Thus Auden felt the pressure of the necessity of doing what he could to avert the war. " (Spender, 1964, p. 32). Sans souscrire à l'idée que l'écrivain doit assumer un rôle politique, Auden estime que la tâche du poète est " d'étendre notre connaissance du Bien et du Mal " et " peut-être de rendre plus urgent le besoin de l'action et de faire apparaître plus clairement sa nature " (cité par Jolicœur, 1983, p. 63). "The Quarry" dont les images entrent en résonance avec celle de "The Three Companions" en fournit une illustration très claire :

$O$ what is that sound which so thrills the ear

Down in the valley drumming, drumming?

Only the scarlet soldiers, dear,

The soldiers are coming.<smiles>[C]1C[Al]C1</smiles>

$\mathrm{O}$ where are you going? Stay with me here!

Were the vows you swore deceiving, deceiving?

No, I promised to love you, dear,

But I must be leaving.

$O$ it's broken the lock and splintered the door,

$O$ it's the gate where they are turning, turning;

Their boots are heavy on the floor

And their eyes are burning.

Les images du poème "The Three Companions" sont prémonitoires. Les fourneaux qui empoisonnent la vallée de leurs fumées délétères sont peut-être moins le symbole de l'activité industrielle qu'une allégorie de la géhenne qui évoque la première guerre mondiale et annonce la suivante, profilant ses fours crématoires. L'allégorie médiévale qui charpente le poème donne une résonance particulière à l'image de la tache sur la peau, signe de la maladie à combattre. Cette tache évoque la peste, spécifiquement la «peste brune " qui est l'autre nom du fascisme. L'odeur de la mort, évoquée au milieu d'une imagerie médiévale qui rappelle l'exposition des cadavres (pendus et gibets au carrefour des chemins), c'est aussi, à ce moment de l'histoire européenne, l'odeur des cadavres sur les champs de bataille et dans les charniers. "Yonder's the midden whose odours will madden " rappelle dans l'évocation de la folie meurtrière ces autres vers du 
poème « On September 1, 1939 », date de la déclaration de la Seconde Guerre mondiale :

The unmentionable odour of death

Offends the September night.

Les poèmes écrits par Auden dans le contexte de la guerre d'Espagne et de Munich valaient aussi pour le totalitarisme stalinien. Ils restent d'actualité devant les horreurs du Kosovo, du Rwanda, du Timor, de la Sierra Leone ou de la Tchétchénie : "Poetry isn't reportage, and its news should be of a permanent significance " (Brodsky, 1986 [1984], p. 353). Au-delà des frontières et du temps, l'indétermination sémantique du poème est ce qui le rend disponible pour tous ces investissements, sans qu'on puisse jamais lui attribuer d'interprétation particulière.

\section{Le projet de la traduction}

Le poème d'Auden ébranle le discours de la méthode qui a cours en traduction. Ici, l'objet du traduire n'est pas (ne peut pas être) ce que le texte "veut dire" ou, à travers lui, ce que son auteur a voulu dire, même si c'est cela aussi. Au texte, lieu problématique d'un "vouloir dire " non moins problématique, on pourrait opposer le projet du texte comme matière de traduction. Le projet du texte est ce qui dispose le texte à signifier, sa signifiance, son dispositif d'écriture.

Ce qui me paraît essentiel dans "The Three Companions", c'est le heurt dialectique érigé en principe d'organisation : la forme euphorique opposée au contenu dysphorique et l'allégorie médiévale qui demeure non résolue (forme connue, sens indécidable). Dans ce dispositif s'u abyment " (héraldiquement) les constituants du poème : structuration ternaire (trinitaire) allant du niveau des strophes jusqu'à celui des phonèmes en passant par la syntaxe et le lexique, et organisation sérielle des personnages allégoriques, de l'espace et du temps; structuration binaire du dialogue encadrant la dualité des personnages allégoriques et les redoublements phonétiques (effet d'écho). A partir de là, j'esquisse deux traductions que je vais opposer pour ne retenir que la seconde. 


\section{DE LA TRADUCTION $1 \ldots$}

\section{Les trois compagnons}

«Où vas-tu donc ainsi? demanda le lecteur Dans la vallée des fours crachent la mort De la fosse montent là-bas d'insanes odeurs Dans le creux de la combe retombent les forts. ”

"Oh! ne crois-tu pas », demanda la peur, Qu'en allant au pertuis la trompeuse nuit Á ton œil éveillé la faille cachera, Et de la pierre au pré te précipitera?»

"Quel était cet oiseau?, demanda l'horreur Et cette forme apparue dans les arbres tordus? Silhouette furtive en silence te guette La tache sur ta peau! Un mal te possède. »

"Loin du foyer » - répondit le chevalier " Jamais au tien » - répondit le pèlerin "Est venue ton heure " - répondit le lecteur Et les autres, les autres quitta.

\section{...À LA TRADUCTION 2}

\section{Les trois compères}

Où vas-tu donc ainsi? demanda le lecteur Dans la vallée des fours donnent la mort Donnent la mort

De la fosse montent là-bas de folles odeurs De folles odeurs Le creux de la combe du preux est la tombe Du preux est la tombe.

Oh! ne crois-tu pas, demanda la peur, Qu'en allant au pertuis la trompeuse nuit La trompeuse nuit

À ton cil vigilant cachera le vide

Cachera le vide

Et de la pierre au pré seras précipité?

Seras précipité.

Quel était cet oiseau? demanda l'horreur Et cette forme apparue dans les arbres tordus? 
Dans les arbres tordus

L'ombre furtive te suit en silence

Te suit en silence

La tache sur ta peau est une pestilence

Est une pestilence.

Loin du foyer - répondit le chevalier

Jamais au tien - répondit le pèlerin

Est venue ton heure - répondit le lecteur

Et les autres quitta, les autres quitta.

La dialectique du texte original est ancrée dans un intertexte resté actuel dans la culture anglo-saxonne, mais pas dans l'espace francophone. La copie des structures phonétiques, aussi ingénieuse soit-elle, ne suffit pas à provoquer la déstabilisation entre forme et contenu d'où le poème tire sa modernité. La reprise appliquée des signes du poème produit au contraire de la vétusté. Transposées en français, les contraintes sonores donnent à l'ensemble un tempo artificiel, sans parler des contorsions de la syntaxe. Qu'on la rapporte au champ de la poésie française ou à l'esthétique d'Auden, la première traduction n'est pas poétiquement plausible. Brodsky aurait une règle pour cela : « In the beginning of every poem, a poet has to dispel that air of art and artifice that clouds the public's attitude to poetry. He has to be convincing, plain - the way, presumably, the public itself is. $\mathrm{He}$ has to speak with a public voice, and all the more so if it is a public subject that he deals with. " $(1986$, p. 310$)$ La première traduction détonne par rapport à l'écriture matter-of-fact d'Auden, au ton dégagé de ses phrases: "That valley is fatal when furnaces burn ", "The spot on your skin is a shocking disease ». Mais sous son apparente simplicité, ce langage ne se confond pas avec le langage ordinaire. $\grave{A}$ tous les niveaux l'insolite voisine avec le familier, donnant au poème son inquiétante étrangeté.

La seconde traduction propose une solution au problème de l'intertexte. Si la ballade en tant que forme fixe est tombée dans l'oubli, la chanson à répondre existe toujours dans l'expression populaire. On peut donc l'investir d'un contenu dysphorique pour provoquer le même choc dialectique, la même " perte d'aura " que l'original. La répétition est un procédé fréquent chez Auden, sans oublier que le modèle immédiat du poème est aussi une chanson à répondre. Cette forme dialoguée se prête à merveille au jeu des répétitions binaires et ternaires. Elle fait bon ménage avec le beat d'Auden. Elle l'amplifie, ou plutôt elle en déplace les points d'appui, cassant ainsi l'effet " mirliton » qui résulte en français de la copie du tétramètre. Comme la répétition introduit une rime régulière, elle donne au traducteur plus de latitude pour simplifier le langage et sa tonalité.

Pour que l'intertexte remplisse la même fonction ludique que dans l'original, on peut employer ce qui le charpente et sert en même 
temps à crypter le sens du poème : la structure en écho et le chiffre trois, le chiffre de la mort. C'est ce qui a déterminé ici le choix et le lieu des répétitions. Pour maintenir la " perte d'aura ", on pourrait déjouer la structure ternaire de ces répétitions : le redoublement qui survient trois fois dans chaque strophe pourrait être brisé tantôt par un chiasme ( " masquera la faille »/" la faille masquera »), tantôt par l'introduction d'un élément nouveau qui correspondrait à la "chute » de l'histoire racontée dans chaque strophe (" Et de la pierre au pré, de la pierre au pré/Seras précipité »). Mais cette option est écartée quand elle réintroduit l'artifice.

Après la forme fixe du poème, l'obstacle le plus difficile à vaincre est celui des paronomases - reader/rider // fearer/farer // horror/hearer - d'autant plus que le fonctionnement allégorique du poème en dépend. Une éternité, en effet, n'y suffirait pas, puisque chacune de ces formes-sens est cadenassée par l'anglais. Trouver, dans la langue française, une équivalence phono-sémantique pour le réseau complexe des six personnages est une entreprise vouée de toute évidence à l'échec. Mais puisque l'appariement des personnages dépend aussi de la structure dialogique du poème, pourquoi ne pas les découpler en ouvrant la forme du dialogue (demanda $\mathrm{x} /$ répondit y)? La structure en écho (reader/rider, etc.) est déplacée dans la troisième strophe sous la forme d'une rime interne (foyer/chevalier, etc.).

L'allégorie s'inscrit dans un réseau sémantique plus vaste, celui de l'isotopie médiévale. Celle-ci englobe la forme du poème (ballade, métrique, dialogue...), les personnages emblématiques et l'imagerie. Cette isotopie détermine le choix du nom de chaque personnage. C'est pourquoi, influencée par la gravure de Dürer et par une ballade anonyme qui figure dans l'anthologie d'Auden (" The Fause Knicht upon the Road ") ${ }^{6}$, j'ai opte pour " chevalier », au lieu de " cavalier», et aussi pour " pèlerin ». La forme chantée du poème m'a soufflé le mot " compère 》 à la place de "compagnon " - ce dernier terme laisse ouverte l'allusion aux "compagnons de route ", mais cet élément est secondaire et le thème du voyage peut l'activer.

Il reste la question du " lecteur», personnage clé, ou plus exactement de son vis-à-vis, "hearer", celui qui occupe la place diamétralement opposée à "reader» dans le poème. Mon optionreprendre le même terme ("lecteur») au lieu d'u oreille» ou " auditeur ") - s'appuie sur la réversibilité des positions dans ce dialogue et plus largement dans le système poétique d'Auden. Cette réversibilité crée dans le texte original un programme de lecture en boucle, une des sources de l'indétermination sémantique qui donne au poème sa force cognitive paradoxale et sa modernité. Même si la

6 "O whare are ye gaun?" / Quo the fause knicht upon the road; / «I am gaun to the scule, "/ Quo' the wee boy, and still he stude. " (Auden, 1973 [1938], p. 98). 
répétition lecteur/lecteur dévoile quelque chose, elle ne pointe jamais que le dispositif d'écriture du poème. Elle montre la boucle, mais la boucle est justement ce qui piège l'interprétation.

L'isotopie médiévale suscite des choix comme celui du mot " preux " (tall) de la première strophe. La préciosité du terme est rachetée par une continuité oppositive, sémantique et phonétique (par dissimilation), avec la "peur » du vers suivant. Le mot " preux » est mis dans la bouche de " reader 》 dont les arguments s'appuient sur une imagerie tirée tout droit des Écritures ("valley ", " furnaces", " burn ", " where the tall return »). " Reader » est le savant, celui qui possède la connaissance, une connaissance livresque des choses. C'est pourquoi j'ai maintenu dans son discours des tournures moins ordinaires.

D'autres termes ("fours ", "fosse ") arrêtés eux aussi pour leur brièveté et leur sonorité tirent le poème vers des événements contemporains et le rendent plus transparent que l'original. Ces termes ont aussi le défaut d'estomper les allusions directes aux Écritures. Dans le même ordre d'idées, l'adjectif «brune " a surgi spontanément pour qualifier « la tache sur ta peau », mais je l'ai vite abandonné au profit d'une formulation qui cadre mieux avec l'image de la maladie qu'Auden utilise pour critiquer l'Angleterre : " La tache sur ta peau est une pestilence ". Au sens figuré, "pestilence » est, selon Littré, un " terme d'Écriture et de théologie qui désigne une mauvaise doctrine ». Ce terme reste donc cohérent dans l'imagerie biblique du poème et par rapport à la "morale " de la fable (si morale il y a). Le rythme et la sonorité encore soufflent des solutions qui font problème. Par exemple, " mal » à la place du terme plus neutre de «maladie »: l'allégorie est sauve, mais le mot appelle une résolution métaphysique qui excède la métaphore plus terre-à-terre du vers original. D'où la préférence accordée à " pestilence ". Même remarque pour le mot « pertuis 》 dont le sens moderne et courant relève du domaine maritime, tandis qu'on active ici le sens latin et un usage régional dans lequel ce mot désigne le "passage d'un versant à un autre " (Littré). La prédilection d'Auden pour les mots anciens ou inusités peut aussi justifier ce choix, comme il justifierait celui d'« insanes» (odeurs) pour faire pendant à l'improbable « midden ».

Je ne suis pas poète, et les longues années occupées à traduire les débats du Parlement canadien ne m'ont pas bien préparée à traduire de la poésie. Qu'on veuille bien me pardonner cette exception (je jure de ne pas récidiver). Pour les besoins de l'exercice qui nous occupe ici, je m'autoriserai à conclure par une dernière observation. La seconde traduction me paraît actualiser mieux que la première le projet du poème. Sa forme proliférante n'est pas fortuite. Elle est inspirée par d'autres œuvres d'Auden. Elle est inspirée aussi par Antonin Artaud 
traduisant la logique du nonsense de Lewis Carroll (1956). ${ }^{7}$ Elle s'inspire également de Cortanze qui exploite le dispositif " créationniste " de Huidobro dans sa traduction-transformations d'Altazor (recueil de poèmes écrits entre 1919 et 1929): " tous les textes présentés ici ne sont pas "essentiels" mais plutôt destinés à montrer ce qui dans une écriture la multiplie " (1976, p. 14).

Serge Gavronsky prend le parti inverse en réduisant chaque vers du poème d'Auden aux signes qui lui sont essentiels et qui fonctionnent alors comme indices. C'est une autre façon d'actualiser le projet cryptogrammatique du poème :

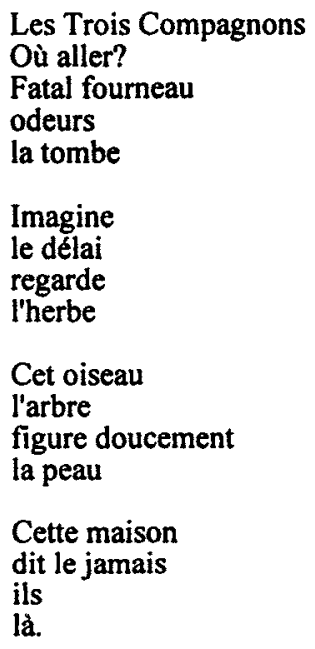

Recomposé de cette façon minimaliste le poème garde sa potentialité assomptive. Il est certain que cette traduction à contre-sens, qui prend les signes à revers (" a contra senso de la pela "), ne permet pas que le poème s'engage dans la voie mitoyenne de l'artifice et du référentiel.

Le projet du texte est le foyer qui rassemble les modalités cognitives du poème, suscite la possibilité d'une assomption de connaissance. Dans La Traduction-poésie, ouvrage dédié à Antoine Berman, Jacques Ancet en résume la conséquence pour la traduction: " Le traducteur ne peut donc être ni un "passeur" ni une

7 Artaud traduit le celebre chapitre "Humpty Dumpty" de Through the Looking Glass en incorporant aux modes de signification du texte de Carroll sa propre conception de la poésie et du rapport somatique à la langue. Cette démarche fusionne l'indétermination créé par Carroll et celle qu'Artaud engendre lui-même. L'une devient ainsi le vecteur de l'autre, au lieu de l'annuler ou d'être annulée par elle. (A. Brisset, 1985) 
entreprise d'import-export. L'opération à laquelle il doit se livrer [...] est beaucoup plus inquiétante : tuer l'original pour le ressusciter dans son propre texte. [...] Mais attention : le corps ressuscité n'est plus le même. Il est d'une autre nature. Ainsi pour le texte - et pour le sens. ” (1999, p. 178).

Université d'Ottawa

\section{Références}

ANCET, Jacques (1999). "La séparation ", Martine Broda (éd.), La Traduction-poésie. Strasbourg, Presses de l'Université de Strasbourg, pp. 173-186.

ARTAUD, Antonin (1956). "L'Arve et l'Aume. Tentative antigrammaticale contre Lewis Carroll ». CEuvres complètes. Paris, Gallimard.

AUDEN, Wystan Hugh (1932). The Orators. Londres, Faber.

-(1941). The Double Man. New York, Random House.

- (1941). New Year Letter. Londres, Faber.

- (1968). Secondary Worlds. Londres, Faber.

- (1973 [1938]). The Oxford Book of Light Verse. London, Oxford University Press.

BACHELARD, Gaston (1948). La Terre et les rêveries de la volonté. Paris, Corti.

BENJAMIN, Walter (1970 [1955]). Charles Baudelaire. Un poète lyrique à l'apogée du capitalisme. Paris, Payot.

BRISSET, Annie (1985). "Antonin Artaud de l'autre côté du miroir: analyse d'une traduction paradoxale ". RSSI (Recherches Sémiotiques/Semiotic Inquiry). Vol. 5, no 2, pp. 129-144.

BRODSKY, Joseph (1986 [1984]). "On "September 1, 1939" by W. H. Auden ». Less than One. Selected Essays by Joseph Brodsky. New York, Farrar Strauss Giroux/Toronto, Colins, pp. 304-356.

DEANE, Patrick (1991). "Within a field that never closes": the reader in W. H. Auden's "New Year Letter" ". Contemporary Literature. Vol. 32, no 2, pp. 171-193. 
HUIDOBRO, Vicente (1976). Manifestes. Altazor. (transformation.s, G. De Cortanze), Bruxelles, Champ Libre.

DURAND, Gilles (1963). Les Structures anthropologiques de l'imaginaire. Paris, Presses universitaires de France.

JOLICCEUR, Claude (1983). " Du goût pour les idéologies durant les années trente ». Textes et langages. $\mathrm{N}^{\circ}$ 9, pp. 53-87.

GREENBERG, Herbert (1968). Quest for the Necessary. W. H. Auden and the Dilemma of Divided Consciousness. Cambridge, Mass., Harvard University Press.

SPENDER, Stephen. (1964). "W. H. Auden and His Poetry ». M. K. Spears (ed.), Auden. A Collection of Critical Essays. Englewood Cliffs, N. J., Prentice-Hall.

WESKER, Arnold (1978). Said the Old Man to the Young Man. Londres, Jonathan Cape.

- (1976). "Chips with Everything 》. The Plays of Arnold Wesker. New York, Harper \& Row, pp. 299-358.

RÉSUMÉ : Traduire le texte dans son projet. Le littéralisme est soluble dans la poésie moderne : Auden - Bien qu'il soit reconnu comme l'un des plus grands poètes du XX' $\mathrm{XX}^{\mathbf{e}}$ siècle, W. H. Auden est peu traduit en français. Son œuvre, il est vrai, résiste à la traduction lorsqu'elle s'amuse, comme dans le poème qui sert ici d'exemple, à crypter le langage de tous les jours en le coulant dans une forme contrainte par la tradition. "The Three Companions 》 est un poème clé dans l'œuvre d'Auden qui le publie au début des années trente et le reconduit dans plusieurs de ses recueils. Nous en proposons une analyse qui ouvre un dialogue avec chacune des traductions (une dizaine) et avec les commentaires qui les accompagnent.

ABSTRACT : Translating the Text in the Context of its Project. Literalism is Soluble in Modern Poetry : Auden - Though he is recognized as one of the great poets of the $20^{\text {th }}$ century, W. H. Auden has been translated very little into French. It is true that his work resists translation when it seems to almost delight in the encryption of everyday language into a form constrained by tradition, as in the poem discussed in this article. "The Three Companions", a key poem in the body of Auden's work, was first published in the early Thirties, and reappeared in many of his subsequent collections of poetry. In this article we propose an analysis reopening the dialogue with the various translations of the poem (10 in all), with their accompanying comments. 
Annie Brisset : École de traduction et d'interprétation, Université d'Ottawa, C.P. 450, Succ. A, Ottawa (Ontario) K1N 6N5

Courriel : abrisset@uottawa.ca 